JouRnal of Universal Mathematics

Vol.1 No.3 PP.293-311 (2018)

ISSN-2618-5660

\title{
EXISTENCE RESULTS FOR AN IMPULSIVE FRACTIONAL INTEGRO-DIFFERENTIAL EQUATIONS WITH A NON-COMPACT SEMIGROUP
}

\author{
K. HILAL, K. GUIDA, L. IBNELAZYZ, AND M. OUKESSOU
}

\begin{abstract}
In this paper we study a fractional differential equations problem with not instantaneous impulses involving a non-compact semigroup. We present some concepts and facts about the strongly continuous semigroup and the measure of noncompactness. After that we give an existence theorem of our problem using a condensing operator and the measure of noncompactness.
\end{abstract}

\section{INTRODUCTION}

The concept of fractional differential equations has become more popular among mathematicians, and is studied extensively in the recent years for its many applications, for more details about fractional differential equations we refer the readers to $[13,14,9,16,17,22,11]$.

Furthermore, impulsive differential equations have known rapid growth because they play a main role in describing modern problems in fields such as physics, biology, economics and population dynamics; for more details the reader can see $[15,1,7]$.

A lot of models about fractional impulsive differential equations were studied recently, for more details we give the references $[19,12,21]$ and the references therein.

In [3] P. Chen, X. Zhang and Y. Li studied the existence of mild solutions for the initial value problem

$$
\left\{\begin{array}{l}
x^{\prime}(t)+A x(t)=f(t, x(t)), t \in\left(s_{i}, t_{i+1}\right], i=0,1,2, \ldots, m, \\
x(t)=g_{i}(t, x(t)), t \in\left(t_{i}, s_{i}\right], i=1,2, \ldots, m, \\
x(0)=x_{0} .
\end{array}\right.
$$

Where $A: D(A) \subset E \longrightarrow E$ is a closed linear operator, $-A$ is the infinitesimal generator of a strongly continuous semigroup $(T(t))_{t \geq 0}$ in $E$, here the semigroup $(T(t))_{t \geq 0}$ is non-compact.

Motivated by this work, in this article we study the impulsive fractional evolution equation

Date: Accepted October 23, 2018.

Key words and phrases. Fractional impulsive differential equations, measure of noncompactness, Darbo-Sadovskii's fixed point theorem. 


$$
\left\{\begin{array}{c}
{ }^{c} D^{\alpha} x(t)=A x(t)+f(t, x(t), B x(t))+C(t) u(t), t \in\left(s_{i}, t_{i+1}\right], \\
i=0,1,2, \ldots, m, u \in U_{a d}, \\
{ }^{c} D^{\beta} x(t)=g_{i}(t, x(t)), t \in\left(t_{i}, s_{i}\right], i=1,2, \ldots, m, \\
x(0)=x_{0},
\end{array}\right.
$$

involving ${ }^{c} D^{\alpha}$ and ${ }^{c} D^{\beta}$ which are the Caputo fractional derivatives of order $\alpha \in$ $(0,1)$ and $\beta \in(0,1)$ respectively with the lower limit zero, $A: D(A) \subset X \longrightarrow X$ is the generator of a non-compact $C_{0}$-semigroup of bounded operators $(T(t))_{t \geq 0}$ on a Banach space $(X,\|\cdot\|), x_{0} \in X, 0=t_{0}=s_{0}<t_{1}<s_{1}<t_{2}<s_{2}<\ldots<t_{m} \leq$ $s_{m}<t_{m+1}=T$ are fixed numbers, $g_{i} \in C(J \times X, X)$, and $B x(t)=\int_{0}^{t} B(t, s) x(s) d s$, $B \in C\left(D_{1}, \mathbb{R}^{+}\right)$, with $D_{1}=\left\{(t, s) \in \mathbb{R}^{2}: 0 \leq s \leq t \leq \omega\right\}, K_{0}=\max _{(t, s) \in D_{1}} B(t, s)$ and $U_{a d}$ is a set that will be defined later.

The rest of the paper is organized as follows. In section 2 we present the notations, definitions and preliminary results needed in the following sections. In section 3, a suitable concept of PC-mild solutions for our problems is introduced. Section 4 is concerned with the existence results of our problems.

\section{Preliminaries AND notations}

Let us set $J=[0, T], J_{0}=\left[0, t_{1}\right], J_{1}=\left(t_{1}, t_{2}\right], \ldots, J_{m-1}=\left(t_{m-1}, t_{m}\right], J_{m}=\left(t_{m}, t_{m+1}\right]$ and introduce the space $P C(J, X):=\left\{x: J \rightarrow X: x \in C\left(\left(t_{i}, t_{i+1}\right], X\right), i=\right.$ $0,1, \ldots, m$ and there exist $x\left(t_{i}^{-}\right)$and $x\left(t_{i}^{+}\right), i=1, \ldots, m$ with $\left.x\left(t_{i}^{-}\right)=x\left(t_{i}^{+}\right)\right\}$. It is clear that $P C(J, X)$ is a Banach space with the norm $\|u\|_{P C}=\sup \{\|u(t)\|: t \in J\}$. Let $Y$ be a separable reflexive Banach space where controls $u$ takes values, and $P_{f}(Y)$ is a class of nonempty closed and convex subsets of $Y$. We suppose that the multivalued map $w:[0, a] \longrightarrow P_{f}(Y)$ is measurable, $w(.) \subset E$, where $E$ is bounded set of $Y$, and the admissible control set $U_{a d}=\left\{u \in L^{p}(E): u(t) \in w(t), a . e\right\}, p>$ $\frac{1}{\tau},(\tau \in(0, \alpha))$, for more details about admissible control set, we refer the readers to $[6]$.

Let us recall the following well-known definitions.

Definition 2.1. [17] The Riemann-Liouville fractional integral of order $q$ with lower limit zero for a function $\mathrm{f}$ is defined as

$$
I^{q} f(t)=\frac{1}{\Gamma(q)} \int_{0}^{t}(t-s)^{q-1} f(s) d s, q>0,
$$

provided the integral exists, where $\Gamma($.$) is the gamma function.$

Definition 2.2. [17] The Riemann-Liouville derivative of order $q$ with the lower limit zero for a function $f:[0, \infty) \rightarrow \mathbb{R}$ can be written as

$$
{ }^{L} D^{q} f(t)=\frac{1}{\Gamma(n-q)} \frac{d^{n}}{d t^{n}} \int_{0}^{t}(t-s)^{n-q-1} f(s) d s, n-1<q<n, t>0 .
$$

Definition 2.3. [17] The Caputo derivative of order q for a function $f:[0, \infty) \longrightarrow$ $\mathbb{R}$ can be written as 


$$
{ }^{c} D^{q} f(t)={ }^{L} D^{q}\left(f(t)-\sum_{k=0}^{k} \frac{t^{k}}{k !} f^{k}(0)\right), n-1<q<n, t>0 .
$$

Definition 2.4. [21] A function $x \in C(J, X)$ is said to be a mild solution of the following problem:

$$
\left\{\begin{array}{l}
{ }^{c} D^{\alpha} x(t)=A x(t)+y(t), t \in(0, T] \\
x(0)=x_{0}
\end{array}\right.
$$

if it satisfies the integral equation

$$
x(t)=P_{\alpha}(t) x_{0}+\int_{0}^{t}(t-s)^{\alpha-1} Q_{\alpha}(t-s) y(s) d s,
$$

Here

$$
\begin{aligned}
P_{\alpha}(t) & =\int_{0}^{\infty} \xi_{\alpha}(\theta) T\left(t^{\alpha} \theta\right) d \theta, Q_{\alpha}(t)=\alpha \int_{0}^{\infty} \theta \xi_{\alpha}(\theta) T\left(t^{\alpha} \theta\right) d \theta, \\
\xi_{\alpha}(\theta) & =\frac{1}{\alpha} \theta^{-1-\frac{1}{\alpha}} \bar{\omega}_{\alpha}\left(\theta^{-\frac{1}{\alpha}}\right) \geq 0, \\
\overline{\omega_{\alpha}}(\theta) & =\frac{1}{\pi} \sum_{n=1}^{\infty}(-1)^{n-1} \theta^{-n \alpha-1} \frac{\Gamma(n \alpha+1)}{n !} \sin (n \pi \alpha), \theta \in(0, \infty), \text { and } \xi_{\alpha}(\theta) \geq 0, \\
\theta \in(0, \infty), & \int_{0}^{\infty} \xi_{\alpha}(\theta) d \theta=1 .
\end{aligned}
$$

It is easy to verify that $\int_{0}^{\infty} \theta \xi_{\alpha}(\theta) d \theta=\frac{1}{\Gamma(1+\alpha)}$.

Theorem 2.5. (Darbo-Sadovskii [8]) if $D \subset X$ is bounded, closed and convex, the continuous map $S: D \rightarrow D$ is $\beta$ - condensing, then $S$ has a fixed point in $D$.

Lemma 2.6. $[4,5,20]$ The operators $P_{\alpha}$ and $Q_{\alpha}$ have the following properties:

(1) For any fixed $t \geq 0, P_{\alpha}(t)$ and $Q_{\alpha}(t)$ are linear and bounded operators, and for any $x \in X$,

$$
\left\|P_{\alpha}(t) x\right\| \leq M_{A}\|x\|,\left\|Q_{\alpha}(t) x\right\| \leq \frac{\alpha M_{A}}{\Gamma(1+\alpha)}\|x\|,
$$

(2) $\left\{P_{\alpha}(t), t \geq 0\right\}$ and $\left\{Q_{\alpha}(t), t \geq 0\right\}$ are strongly continuous,

(3) if $(T(t))_{t \geq 0}$ is an equicontinuous semigroup, then $P_{\alpha}(t)$ and $Q_{\alpha}(t)$ are continuous in $(0, \infty)$ by the norm, which means that for $0<t^{\prime}<t^{\prime \prime}<T$ we have:

$$
\left\|P_{\alpha}\left(t^{\prime \prime}\right)-P_{\alpha}\left(t^{\prime}\right)\right\| \longrightarrow 0 \text { and }\left\|Q_{\alpha}\left(t^{\prime \prime}\right)-Q_{\alpha}\left(t^{\prime}\right)\right\| \longrightarrow 0 \text { as } t^{\prime \prime} \longrightarrow t^{\prime} .
$$

Definition 2.7. [8] Let $X$ be a Banach space and $\Omega_{X}$ be the bounded subsets of X. The Kuratowski measure of noncompactness is the map $\beta: \Omega_{X} \longrightarrow[0, \infty)$ defined by :

$\beta(B)=\inf \left\{\epsilon>0: B=\cup_{i=1}^{n} B_{i}\right.$ and $\operatorname{diam}\left(B_{i}\right) \leq \epsilon$ for $\left.i=1,2, . ., n\right\}$ with $\operatorname{diam} B_{i}=\sup \left\{|x-y|: x, y \in B_{i}\right\}$ and $B \in \Omega_{X}$

Remark 2.8. It is clear that $\beta(B) \leq \operatorname{diam}(B)$.

Next, we are going to look back on some properties of the measure of noncompactness that will be used in the proof of our main results.

Lemma 2.9. [8] Let $A$ and $B$ be bounded sets of $X$ and $\lambda$ be a real number. Then the measure of noncompactness has the following properties:

1) $\beta(A)=0$ if and only if $A$ is a relatively compact set,

2) $A \subset B$ implies that $\beta(A) \leq \beta(B)$, 
3) $\beta(\bar{A})=\beta(A)$,

4) $\beta(A \cup B)=\max \{\beta(A), \beta(B)\}$,

5) $\beta(\lambda A)=|\lambda| \beta(A)$,

6) $\beta(A+B) \leq \beta(A)+\beta(B)$,

7) $\beta(\overline{c o} A)=\beta(A)$.

Where $\overline{c o}$ means the closure of the convex hull.

Lemma 2.10. [2] Let $X$ be a Banach space, $W \subset X$ be bounded. Then there exists a countable set $W_{1} \subset W$ such that

$$
\beta(W) \leq 2 \beta\left(W_{1}\right) .
$$

Lemma 2.11. [8] Let $X$ be a Banach space, $W \subset C(J, X)$ be bounded and equicontinuous. Then $\beta(W(t))$ is continuous on $J$ and

$$
\beta(W)=\max _{t \in J} \beta(W(t))=\beta(W(J)) .
$$

Lemma 2.12. [10] Let $X$ be a Banach space, $W=\left\{u_{n}\right\} \in C(J, X)$ be bounded and countable set. Then $\beta(W(t))$ is a Lebesgue integral on $J$ and

$$
\beta\left(\left\{\int_{J} u_{n}(t) d t: n \in \mathbb{N}\right\}\right) \leq 2 \int_{J} \beta(W(t)) d t .
$$

\section{The CONSTRUCtion OF MiLd SOLUtions}

Let $x \in P C(J, X)$. We first consider the following fractional impulsive problem:

$\left\{\begin{array}{l}{ }^{c} D^{\alpha} x(t)=A x(t)+f(t, x(t), B x(t))+C(t) u(t), t \in\left(s_{i}, t_{i+1}\right], i=0,1,2, \ldots, m, u \in U_{a d}, \\ { }^{c} D^{\beta} x(t)=g_{i}(t, x(t)), t \in\left(t_{i}, s_{i}\right], i=1,2, \ldots, m, \\ x(0)=x_{0} .\end{array}\right.$

From the property of the Caputo derivative, a general solution can be written as

$x(t)=\left\{\begin{array}{l}x_{0}+\frac{1}{\Gamma(\alpha)} \int_{0}^{t}(t-s)^{\alpha-1}[A x(s)+f(s, x(s), B x(s))+C(s) u(s)] d s, t \in\left(0, t_{1}\right], \\ d_{1 x}+\frac{1}{\Gamma(\beta)} \int_{0}^{t}(t-s)^{\beta-1} g_{1}(s, x(s)) d s, t \in\left(t_{1}, s_{1}\right], \\ K_{1 x}+\frac{1}{\Gamma(\alpha)} \int_{0}^{t}(t-s)^{\alpha-1}[A x(s)+f(s, x(s), B x(s))+C(s) u(s)] d s, t \in\left(s_{1}, t_{2}\right], \\ \cdot \\ d_{i x}+\frac{1}{\Gamma(\beta)} \int_{0}^{t}(t-s)^{\beta-1} g_{i}(s, x(s)) d s, t \in\left(t_{i}, s_{i}\right], \\ K_{i x}+\frac{1}{\Gamma(\alpha)} \int_{0}^{t}(t-s)^{\alpha-1}[A x(s)+f(s, x(s), B x(s))+C(s) u(s)] d s, t \in\left(s_{i}, t_{i+1}\right],\end{array}\right.$

where $d_{i x}$ and $K_{i x}, i=1,2, \ldots, m$, are elements of X.

We obtain 
$x(t)=\left\{\begin{array}{l}d_{i x}+\frac{1}{\Gamma(\beta)} \int_{0}^{t}(t-s)^{\beta-1} g_{i}(s, x(s)) d s, t \in\left(t_{i}, s_{i}\right], 1 \leq i \leq m, \\ P_{\alpha}\left(t-s_{i}\right) K_{i x}+\int_{0}^{t}(t-s)^{\alpha-1} Q_{\alpha}(t-s)\left[\begin{array}{r}f(s, x(s), B x(s))+C(s) u(s)] d s, \\ t \in\left(s_{i}, t_{i+1}\right], 0 \leq i \leq m,\end{array}\right. \\ K_{0 x}=x_{0} .\end{array}\right.$

And using the fact that $x$ is continuous at the points $t_{i}$, we get :

$$
\begin{aligned}
x\left(t_{i}\right) & =P_{\alpha}\left(t_{i}-s_{i-1}\right) K_{(i-1) x}+\int_{0}^{t_{i}}\left(t_{i}-s\right)^{\alpha-1} Q_{\alpha}\left(t_{i}-s\right)[f(s, x(s), B x(s))+C(s) u(s)] d s \\
& =d_{i x}+\frac{1}{\Gamma(\beta)} \int_{0}^{t_{i}}\left(t_{i}-s\right)^{\beta-1} g_{i}(s, x(s)) d s .
\end{aligned}
$$

Which implies that:

$$
\begin{aligned}
d_{i x}= & P_{\alpha}\left(t_{i}-s_{i-1}\right) K_{(i-1) x}+\int_{0}^{t_{i}}\left(t_{i}-s\right)^{\alpha-1} Q_{\alpha}\left(t_{i}-s\right)[f(s, x(s), B x(s))+C(s) u(s)] d s \\
& -\frac{1}{\Gamma(\beta)} \int_{0}^{t_{i}}\left(t_{i}-s\right)^{\beta-1} g_{i}(s, x(s)) d s .
\end{aligned}
$$

Using the fact that $x$ is continuous at the points $s_{i}$, we get :

$$
\begin{aligned}
x\left(s_{i}\right) & =d_{i x}+\frac{1}{\Gamma(\beta)} \int_{0}^{s_{i}}\left(s_{i}-s\right)^{\beta-1} g_{i}(s, x(s)) d s \\
& =K_{i x}+\int_{0}^{s_{i}}\left(s_{i}-s\right)^{\alpha-1} Q_{\alpha}\left(s_{i}-s\right)[f(s, x(s), B x(s))+C(s) u(s)] d s .
\end{aligned}
$$

Which implies that:

$$
\begin{aligned}
K_{i x}= & d_{i x}+\frac{1}{\Gamma(\beta)} \int_{0}^{s_{i}}\left(s_{i}-s\right)^{\beta-1} g_{i}(s, x(s)) d s \\
& -\int_{0}^{s_{i}}\left(s_{i}-s\right)^{\alpha-1} Q_{\alpha}\left(s_{i}-s\right)[f(s, x(s), B x(s))+C(s) u(s)] d s .
\end{aligned}
$$

Therefore, a mild solution of problem (1.2) is given by

$$
x(t)=\left\{\begin{array}{l}
P_{\alpha}(t) x_{0}+\int_{0}^{t}(t-s)^{\alpha-1} Q_{\alpha}(t-s)[f(s, x(s), B x(s))+C(s) u(s)] d s, t \in\left(0, t_{1}\right], \\
d_{1}+\frac{1}{\Gamma(\beta)} \int_{0}^{t}(t-s)^{\beta-1} g_{1}(s, x(s)) d s, t \in\left(t_{1}, s_{1}\right], \\
P_{\alpha}\left(t-s_{1}\right) K_{1}+\int_{0}^{t}(t-s)^{\alpha-1} Q_{\alpha}(t-s)[f(s, x(s), B x(s))+C(s) u(s)] d s, t \in\left(s_{1}, t_{2}\right], \\
\cdot \\
\cdot \\
d_{i x}+\frac{1}{\Gamma(\beta)} \int_{0}^{t}(t-s)^{\beta-1} g_{i}(s, x(s)) d s, t \in\left(t_{i}, s_{i}\right], 1 \leq i \leq m, \\
P_{\alpha}\left(t-s_{i}\right) K_{i x}+\int_{0}^{t}(t-s)^{\alpha-1} Q_{\alpha}(t-s)[f(s, x(s), B x(s))+C(s) u(s)] d s, \\
t \in\left(s_{i}, t_{i+1}\right], 1 \leq i \leq m,
\end{array}\right.
$$

where

$K_{0 x}=x_{0}$, 


$$
\begin{aligned}
d_{i x}= & P_{\alpha}\left(t_{i}-s_{i-1}\right) K_{(i-1) x}+\int_{0}^{t_{i}}\left(t_{i}-s\right)^{\alpha-1} Q_{\alpha}\left(t_{i}-s\right)[f(s, x(s), B x(s))+C(s) u(s)] d s \\
& -\frac{1}{\Gamma(\beta)} \int_{0}^{t_{i}}\left(t_{i}-s\right)^{\beta-1} g_{i}(s, x(s)) d s, \\
K_{i x}= & d_{i x}-\int_{0}^{s_{i}}\left(s_{i}-s\right)^{\alpha-1} Q_{\alpha}\left(s_{i}-s\right)[f(s, x(s), B x(s))+C(s) u(s)] d s \\
& +\frac{1}{\Gamma(\beta)} \int_{0}^{s_{i}}\left(s_{i}-s\right)^{\beta-1} g_{i}(s, x(s)) d s .
\end{aligned}
$$

Definition 3.1. A function $x \in P C(J, X)$ is said to be a mild solution of problem

(1.2) if it satisfies the following relation:

$$
x(t)=\left\{\begin{array}{c}
P_{\alpha}(t) K_{0 x}+\int_{0}^{t}(t-s)^{\alpha-1} Q_{\alpha}(t-s)[f(s, x(s), B x(s))+C(s) u(s)] d s, \\
t \in\left(0, t_{1}\right], u \in U_{a d}, \\
d_{i x}+\frac{1}{\Gamma(\beta)} \int_{0}^{t}(t-s)^{\beta-1} g_{i}(s, x(s)) d s, t \in\left(t_{i}, s_{i}\right], 1 \leq i \leq m, \\
P_{\alpha}\left(t-s_{i}\right) K_{i x}+\int_{0}^{t}(t-s)^{\alpha-1} Q_{\alpha}(t-s)\left[\begin{array}{c}
f(s, x(s), B x(s))+C(s) u(s)] d s, \\
t \in\left(s_{i}, t_{i+1}\right], 1 \leq i \leq m .
\end{array}\right.
\end{array}\right.
$$

Where

$$
\begin{aligned}
K_{0 x}= & x_{0}, \\
d_{i x}= & P_{\alpha}\left(t_{i}-s_{i-1}\right) K_{(i-1) x}+\int_{0}^{t_{i}}\left(t_{i}-s\right)^{\alpha-1} Q_{\alpha}\left(t_{i}-s\right)[f(s, x(s), B x(s))+C(s) u(s)] d s \\
& \quad-\frac{1}{\Gamma(\beta)} \int_{0}^{t_{i}}\left(t_{i}-s\right)^{\beta-1} g_{i}(s, x(s)) d s, \\
K_{i x}= & d_{i x}-\int_{0}^{s_{i}}\left(s_{i}-s\right)^{\alpha-1} Q_{\alpha}\left(s_{i}-s\right)[f(s, x(s), B x(s))+C(s) u(s)] d s \\
& +\frac{1}{\Gamma(\beta)} \int_{0}^{s_{i}}\left(s_{i}-s\right)^{\beta-1} g_{i}(s, x(s)) d s .
\end{aligned}
$$

\section{Existence RESUlts}

This section deals with the existence results of the problem (1.2).

To prove our first existence result we introduce the following assumptions.

$\left(H_{1}\right)$ A generates an equicontinuous and uniformly bounded strongly continuous semigroup $T(t)_{t \geq 0}$ on a Banach space $X$ such that $\|T(t)\| \leq M_{A}$ for all $t \in J$,

$\left(H_{2}\right) C:[0, T] \longrightarrow L(Y, X)$ is essentially bounded, ie $C \in L^{\infty}([0, T], L(Y, X))$,

$\left(H_{3}\right)$ The function $f \in C(J \times X \times X, X)$,

$\left(H_{4}\right)$ there exists $\tau \in(0, \alpha)$ and a positive function $m \in L^{\frac{1}{\tau}}(J, \mathbb{R})$ such that

$\|f(t, u, v)\| \leq m(t)$, for $u, v \in X$ and $t \in J$,

$\left(H_{5}\right)$ For $i=1,2, \ldots, m$, the function $g_{i} \in C\left(\left[t_{i}, s_{i}\right] \times X ; X\right)$ and there exists constants $K_{i}>0$ such that:

$$
\left\|g_{i}(t, u)-g_{i}(t, v)\right\| \leq K_{i}\|u-v\| \text { for all } u, v \in X, \text { and } K=\max _{1 \leq i \leq m}\left\{K_{i}\right\}
$$


$\left(H_{5}^{\prime}\right)$ For $i=1,2, \ldots, m, g_{i} \in C(J \times X ; X)$ is completely continuous, and there exists constants $b, d>0$ such that:

$\left\|g_{i}(t, u)\right\| \leq b_{i}\|u\|+d_{i}$, for all $u \in B_{r} . B_{r}$ is a set that will be defined later, and $b=\max _{1 \leq i \leq m}\left\{b_{i}\right\}, d=\max _{1 \leq i \leq m}\left\{d_{i}\right\}$,

$\left(H_{6}\right)$ There exists constants $L_{1}, L_{2}>0$ such that:

$\beta\left(f\left(t, D_{1}, D_{2}\right) \leq L_{1} \beta\left(D_{1}\right)+L_{2} \beta\left(D_{2}\right)\right.$ for all $t \in J$ and $D_{1}, D_{2}$ bounded and countable sets in $X$.

Theorem 4.1. Assume that $\left(H_{1}\right)-\left(H_{6}\right)$ hold. In addition, let's suppose that the following property is verified:

$$
\max \{A, B\}<1
$$

$$
\begin{aligned}
\text { with } & {\left[\frac{\left(s_{m}^{\beta}+t_{m}^{\beta}\right)+M_{A}\left(s_{m-1}^{\beta}+t_{m-1}^{\beta}\right)+\ldots+M_{A}^{m-1}\left(s_{1}^{\beta}+t_{1}^{\beta}\right)}{\Gamma(\beta+1)}\right] K } \\
& +4 M_{A}\left(L_{1}+\omega L_{2} K_{0}\right)\left[\frac{t_{m}^{\alpha}+M_{A}\left(t_{m-1}^{\alpha}+s_{m-1}^{\alpha}\right)+\ldots+M_{A}^{m-1}\left(t_{1}^{\alpha}+s_{1}^{\alpha}\right)}{\Gamma(\alpha+1)}\right]
\end{aligned}
$$

and

$$
\begin{aligned}
B= & {\left[\frac{M_{A}\left(s_{m}^{\beta}+t_{m}^{\beta}\right)+M_{A}^{2}\left(s_{m-1}^{\beta}+t_{m-1}^{\beta}\right)+\ldots+M_{A}^{m}\left(s_{1}^{\beta}+t_{1}^{\beta}\right)}{\Gamma(\beta+1)}\right] K } \\
& +4 M_{A}\left(L_{1}+\omega L_{2} K_{0}\right)\left[\frac{t_{m+1}^{\alpha}+M_{A}\left(t_{m}^{\alpha}+s_{m}^{\alpha}\right)+\ldots+M_{A}^{m}\left(t_{1}^{\alpha}+s_{1}^{\alpha}\right)}{\Gamma(\alpha+1)}\right]
\end{aligned}
$$

Then the problem (1.2) has at least one mild solution.

Proof. We introduce the composition $Q=Q_{1}+Q_{2}$ where :

$$
\begin{gathered}
Q_{1} x(t)=\left\{\begin{array}{r}
P_{\alpha}(t) x_{0}+\int_{0}^{t}(t-s)^{\alpha-1} Q_{\alpha}(t-s) C(s) u(s) d s, t \in\left[0, t_{1}\right], \\
d_{i 1 x}+\frac{1}{\Gamma(\beta)} \int_{0}^{t}(t-s)^{\beta-1} g_{i}(s, x(s)) d s, t \in\left(t_{i}, s_{i}\right], i=1,2, . ., m, \\
P_{\alpha}\left(t-s_{i}\right) K_{i 1 x}+\int_{0}^{t}(t-s)^{\alpha-1} Q_{\alpha}(t-s) C(s) u(s) d s, \\
t \in\left(s_{i}, t_{i+1}\right], i=1,2, . ., m,
\end{array}\right. \\
Q_{2} x(t)=\left\{\begin{array}{r}
\int_{0}^{t}(t-s)^{\alpha-1} Q_{\alpha}(t-s) f(s, x(s), B x(s)) d s, t \in\left[0, t_{1}\right], \\
d_{i 2 x}, t \in\left(t_{i}, s_{i}\right], i=1,2, . ., m, \\
P_{\alpha}\left(t-s_{i}\right) K_{i 2 x}+\int_{0}^{t}(t-s)^{\alpha-1} Q_{\alpha}(t-s) f(s, x(s), B x(s)) d s, \\
t \in\left(s_{i}, t_{i+1}\right], i=1,2, . ., m,
\end{array}\right.
\end{gathered}
$$

with 


$$
\left\{\begin{aligned}
d_{i 1 x}= & P_{\alpha}\left(t_{i}-s_{i-1}\right) K_{(i-1) 1 x}+\int_{0}^{t_{i}}\left(t_{i}-s\right)^{\alpha-1} Q_{\alpha}\left(t_{i}-s\right) C(s) u(s) d s \\
& -\frac{1}{\Gamma(\beta)} \int_{0}^{t_{i}}\left(t_{i}-s\right)^{\beta-1} g_{i}(s, x(s)) d s, \quad i=1,2, . ., m, \\
K_{i 1 x}= & d_{i 1 x}+\frac{1}{\Gamma(\beta)} \int_{0}^{s_{i}}\left(s_{i}-s\right)^{\beta-1} g_{i}(s, x(s)) d s \\
& -\int_{0}^{s_{i}}\left(s_{i}-s\right)^{\alpha-1} Q_{\alpha}\left(s_{i}-s\right) C(s) u(s) d s, i=1,2, . ., m, \\
K_{01 x}= & x_{0},
\end{aligned}\right.
$$

and

$\left\{\begin{array}{l}d_{i 2 x}=P_{\alpha}\left(t_{i}-s_{i-1}\right) K_{(i-1) 2 x}+\int_{0}^{t_{i}}\left(t_{i}-s\right)^{\alpha-1} Q_{\alpha}\left(t_{i}-s\right) f(s, x(s), B x(s)) d s, i=1,2, . ., m, \\ K_{i 2 x}=d_{i 2 x}-\int_{0}^{s_{i}}\left(s_{i}-s\right)^{\alpha-1} Q_{\alpha}\left(s_{i}-s\right) f(s, x(x), B x(s)) d s, i=1,2, . ., m, \\ K_{02 x}=0 .\end{array}\right.$

Our proof will be divided into several steps.

Step1: We show that $Q B_{r}(J) \subset B_{r}(J)$

where $B_{r}=\{x \in P C(J, X) ;\|x\| \leq r\}$ the ball with radius $r>0$;

$$
\begin{aligned}
& K_{\alpha, \tau}=\left(\frac{1-\tau}{\alpha-\tau}\right)^{1-\tau}\|C u\|_{L^{1 / \tau}} \text { and } S_{\alpha, \tau}=\left(\frac{1-\tau}{\alpha-\tau}\right)^{1-\tau}\|m\|_{L^{1 / \tau}}, \\
& \gamma_{1}=M_{A}^{m+1}\left\|x_{0}\right\| \frac{\Gamma(\beta+1)}{\Gamma(\beta+1)-M_{A} K\left(t_{1}^{\beta}+s_{1}^{\beta}\right)}, \\
& \gamma_{2}=\frac{\left(M_{A} t_{m+1}^{\alpha-\tau}+M_{A}^{2}\left(t_{m}^{\alpha-\tau}+s_{m}^{\alpha-\tau}\right)+\ldots+M_{A}^{m+1}\left(t_{1}^{\alpha-\tau}+s_{1}^{\alpha-\tau}\right)\right) \Gamma(\beta+1)}{\left(\Gamma(\beta+1)-M_{A} K\left(t_{1}^{\beta}+s_{1}^{\beta}\right)\right) \Gamma(\alpha)}\left(K_{\alpha, \tau}+\right.
\end{aligned}
$$

$\left.S_{\alpha, \tau}\right)$

$$
\gamma_{3}=\frac{M_{A}^{2}\left(t_{m-1}^{\beta}+s_{m-1}^{\beta}\right)+\ldots+M_{A}^{m}\left(t_{1}^{\beta}+s_{1}^{\beta}\right)}{\left(\Gamma(\beta+1)-M_{A} K\left(t_{1}^{\beta}+s_{1}^{\beta}\right)\right)}\left\|g_{m}(t, 0)\right\|,
$$

here $\gamma_{1}+\gamma_{1}+\gamma_{1} \leq r$.

For any $x \in B_{r}$, we have:

Case1.For $t \in\left[0, t_{1}\right]$

$$
\begin{aligned}
\|Q x(t)\| & \leq\left\|P_{\alpha}(t) K_{0 x}\right\|+\left\|\int_{0}^{t}(t-s)^{\alpha-1} Q_{\alpha}(t-s)[f(s, x(s), B x(s))+C(s) u(s)] d s\right\| \\
& \leq M_{A}\left\|x_{0}\right\|+\frac{M_{A} t_{1}^{\alpha-\tau}}{\Gamma(\alpha)}\left(K_{\alpha, \tau}+S_{\alpha, \tau}\right) .
\end{aligned}
$$

Case 2.For $t \in\left(t_{i}, s_{i}\right], i=1,2, \ldots, m$.

For $t \in\left(t_{1}, s_{1}\right]$ 


$$
\begin{aligned}
\|Q x(t)\| \leq & \left\|d_{1 x}\right\|+\left\|\frac{1}{\Gamma(\beta)} \int_{0}^{t}(t-s)^{\beta-1} g_{1}(s, x(s)) d s\right\| \\
\leq & \| P_{\alpha}\left(t_{1}\right) K_{0 x} \\
& +\int_{0}^{t_{1}}\left(t_{1}-s\right)^{\alpha-1} Q_{\alpha}\left(t_{1}-s\right)[f(s, x(s), B x(s))+C(s) u(s)] d s \| \\
& +\frac{1}{\Gamma(\beta)}\left\|\int_{0}^{t_{1}}\left(t_{1}-s\right)^{\beta-1} g_{1}(s, x(s)) d s\right\|+\| \frac{1}{\Gamma(\beta)} \int_{0}^{t}(t-s)^{\beta-1} g_{1}(s, x(s) d s \| \\
\leq & M_{A}\left\|x_{0}\right\|+\frac{M_{A} t_{1}^{\alpha-\tau}}{\Gamma(\alpha)}\left(K_{\alpha, \tau}+S_{\alpha, \tau}\right)+\frac{t_{1}^{\beta}+s_{1}^{\beta}}{\Gamma(\beta+1)}\left(K\|x\|+\left\|g_{1}(t, 0)\right\|\right) \\
\leq & r .
\end{aligned}
$$

For $t \in\left(s_{1}, t_{2}\right]$

$$
\begin{aligned}
\|Q x(t)\| \leq & \left\|P_{\alpha}\left(t-s_{1}\right) K_{1 x}\right\|+\left\|\int_{0}^{t}(t-s)^{\alpha-1} Q_{\alpha}(t-s)[f(s, x(s), B x(s))+C(s) u(s)] d s\right\| \\
\leq & \left\|P_{\alpha}\left(t-s_{1}\right) d_{1 x}\right\| \\
& +\| P_{\alpha}\left(t-s_{1}\right)\left(\int_{0}^{s_{1}}\left(s_{1}-s\right)^{\alpha-1} Q_{\alpha}(t-s)[f(s, x(s), B x(s))+C(s) u(s)] d s\right. \\
& \left.+\frac{1}{\Gamma(\beta)} \int_{0}^{s_{1}}\left(s_{1}-s\right)^{\beta-1} g_{1}(s, x(s)) d s\right) \| \\
& +\left\|\int_{0}^{t}(t-s)^{\alpha-1} Q_{\alpha}(t-s)[f(s, x(s), B x(s))+C(s) u(s)] d s\right\| \\
\leq & M_{A}^{2}\left\|x_{0}\right\| \\
& +\frac{M_{A}^{2}\left(t_{1}^{\alpha-\tau}+s_{1}^{\alpha-\tau}\right)+M_{A} t_{2}^{\alpha-\tau}}{\Gamma(\alpha)}\left(K_{\alpha, \tau}+S_{\alpha, \tau}\right) \\
& +\frac{M_{A}\left(t_{1}^{\beta}+s_{1}^{\beta}\right)}{\Gamma(\beta+1)}\left(K\|x\|+\left\|g_{1}(t, 0)\right\|\right) \\
\leq & r .
\end{aligned}
$$

We suppose that: for $1 \leq j \leq i$.

For $t \in\left(t_{j}, s_{j}\right]$

$$
\begin{aligned}
\|Q x(t)\| \leq & M_{A}^{j}\left\|x_{0}\right\|+\frac{M_{A} t_{j}^{\alpha-\tau}+M_{A}^{2}\left(t_{j-1}^{\alpha-\tau}+s_{j-1}^{\alpha-\tau}\right)+\ldots+M_{A}^{j}\left(t_{1}^{\alpha-\tau}+s_{1}^{\alpha-\tau}\right)}{\Gamma(\alpha)}\left(K_{\alpha, \tau}+S_{\alpha, \tau}\right) \\
& +\frac{\left(t_{j}^{\beta}+s_{j}^{\beta}\right)+M_{A}\left(t_{j-1}^{\beta}+s_{j-1}^{\beta}\right)+\ldots+M_{A}^{j-1}\left(t_{1}^{\beta}+s_{1}^{\beta}\right)}{\Gamma(\beta+1)}\left(K\|x\|+\left\|g_{j}(t, 0)\right\|\right)
\end{aligned}
$$

For $t \in\left(s_{j}, t_{j+1}\right]$

$$
\begin{aligned}
\|Q x(t)\| \leq & M_{A}^{j+1}\left\|x_{0}\right\|+\frac{M_{A} t_{j+1}^{\alpha-\tau}+M_{A}^{2}\left(t_{j}^{\alpha-\tau}+s_{j}^{\alpha-\tau}\right)+\ldots+M_{A}^{j+1}\left(t_{1}^{\alpha-\tau}+s_{1}^{\alpha-\tau}\right)}{\Gamma(\alpha)}\left(K_{\alpha, \tau}+S_{\alpha, \tau}\right) \\
& +\frac{M_{A}\left(t_{j}^{\beta}+s_{j}^{\beta}\right)+M_{A}^{2}\left(t_{j-1}^{\beta}+s_{j-1}^{\beta}\right)+\ldots+M_{A}^{j}\left(t_{1}^{\beta}+s_{1}^{\beta}\right)}{\Gamma(\beta+1)}\left(K\|x\|+\left\|g_{j}(t, 0)\right\|\right) \\
\leq & r .
\end{aligned}
$$

And we prove the relations for $j=i+1$.

For $t \in\left(t_{i+1}, s_{i+1}\right]$ 


$$
\begin{aligned}
\|Q x(t)\| \leq & \left\|d_{(i+1) x}\right\|+\| \frac{1}{\Gamma(\beta)} \int_{0}^{t}(t-s)^{\beta-1} g_{i+1}(s, x(s) d s \| \\
\leq & \left.\| P_{\alpha}\left(t_{i+1}-s_{i}\right)\right) K_{i x}+\int_{0}^{t_{i+1}}\left(t_{i+1}-s\right)^{\alpha-1} Q_{\alpha}\left(t_{i+1}-s\right)[f(s, x(s), B x(s))+C(s) u(s)] d s \| \\
& +\| \frac{1}{\Gamma(\beta)} \int_{0}^{t_{i+1}}\left(t_{i+1}-s\right)^{\beta-1} g_{i+1}(s, x(s)) d s \\
& +\| \frac{1}{\Gamma(\beta)} \int_{0}^{t}(t-s)^{\beta-1} g_{i+1}(s, x(s) d s \| \\
\leq & M_{A}^{i+1}\left\|x_{0}\right\|+\frac{M_{A} t_{i+1}^{\alpha-\tau}+M_{A}^{2}\left(t_{i}^{\alpha-\tau}+s_{i}^{\alpha-\tau}\right)+\ldots+M_{A}^{i+1}\left(t_{1}^{\alpha-\tau}+s_{1}^{\alpha-\tau}\right)}{\Gamma(\alpha)}\left(K_{\alpha, \tau}+S_{\alpha, \tau}\right) \\
& +\frac{\left(t_{i+1}^{\beta}+s_{i+1}^{\beta}\right)+M_{A}\left(t_{i}^{\beta}+s_{i}^{\beta}\right)+\ldots+M_{A}^{i}\left(t_{1}^{\beta}+s_{1}^{\beta}\right)}{\Gamma(\beta+1)}\left(K\|x\|+\left\|g_{i+1}(t, 0)\right\|\right) \\
\leq & r .
\end{aligned}
$$

For $t \in\left(s_{i+1}, t_{i+2}\right]$

$$
\begin{aligned}
\|Q x(t)\| & \leq M_{A}^{i+2}\left\|x_{0}\right\|+\frac{M_{A} t_{i+2}^{\alpha-\tau}+M_{A}^{2}\left(t_{i+1}^{\alpha-\tau}+s_{i+1}^{\alpha-\tau}\right)+\ldots+M_{A}^{i+2}\left(t_{1}^{\alpha-\tau}+s_{1}^{\alpha-\tau}\right)}{\Gamma(\alpha)}\left(K_{\alpha, \tau}+S_{\alpha, \tau}\right) \\
& +\frac{M_{A}\left(t_{i+1}^{\beta}+s_{i+1}^{\beta}\right)+M_{A}^{2}\left(t_{i}^{\beta}+s_{i}^{\beta}\right)+\ldots+M_{A}^{i+1}\left(t_{1}^{\beta}+s_{1}^{\beta}\right)}{\Gamma(\beta+1)}\left(K\|x\|+\left\|g_{i+1}(t, 0)\right\|\right) \\
& \leq r .
\end{aligned}
$$

We proved that $Q B_{r}(J) \subset B_{r}(J)$.

Step2: $Q_{1}$ is lipschitz. Let $x, y \in P C(J, X)$,

Case 1. For $t \in\left[0, t_{1}\right]$, we have:

$\left\|Q_{1} x(t)-Q_{1} y(t)\right\|=0$.

Similar to the proof on Step1, we prove that:

Case 2. For $t \in\left[t_{i}, s_{i}\right], 1 \leq i \leq m$,

$\left\|Q_{1} x(t)-Q_{1} y(t)\right\| \leq\left[\frac{\left(s_{i}^{\beta}+t_{i}^{\beta}\right)+M_{A}\left(s_{i-1}^{\beta}+t_{i-1}^{\beta}\right)+\ldots+M_{A}^{i-1}\left(s_{1}^{\beta}+t_{1}^{\beta}\right)}{\Gamma(\beta+1)}\right] K\|x-y\|_{P C}$.

For $t \in\left[s_{i}, t_{i+1}\right], 1 \leq i \leq m$,

$\left\|Q_{1} x(t)-Q_{1} y(t)\right\| \leq\left[\frac{M_{A}\left(s_{i}^{\beta}+t_{i}^{\beta}\right)+M_{A}^{2}\left(s_{i-1}^{\beta}+t_{i-1}^{\beta}\right)+\ldots+M_{A}^{i}\left(s_{1}^{\beta}+t_{1}^{\beta}\right)}{\Gamma(\beta+1)}\right] K\|x-y\|_{P C}$.

This implies that $Q_{1}$ is Lipschitz.

Step3: $Q_{2}$ is continuous. 
Let $\left(x_{n}\right)_{n \geq 0}$ be a sequence such that $\lim _{x \rightarrow \infty}\left\|x_{n}-x\right\|_{P C}=0$, we have :

Case 1. For $t \in\left[0, t_{1}\right]$

$$
\begin{aligned}
\left\|Q_{2} x_{n}(t)-Q_{2} x(t)\right\| & \leq\left\|\int_{0}^{t}(t-s)^{\alpha-1} Q_{\alpha}(t-s)\left(f\left(s, x_{n}(s), B x_{n}(s)\right)-f(s, x(s), B x(s))\right) d s\right\| \\
& \leq \frac{M_{A} t_{1}^{\alpha}}{\Gamma(\alpha+1)}\left\|f\left(., x_{n}(.), B x_{n}(.)\right)-f(., x(.), B x(.))\right\|_{P C} .
\end{aligned}
$$

Similar to the proof we did in Step1, we prove that:

Case 2. For $t \in\left(t_{i}, s_{i}\right], i=1,2, \ldots, m$,

$$
\left\|Q_{2} x_{n}(t)-Q_{2} x(t)\right\| \leq\left[\frac{M_{A} t_{i}^{\alpha}+M_{A}^{2}\left(t_{i-1}^{\alpha}+s_{i-1}^{\alpha}\right)+\ldots+M_{A}^{i}\left(t_{1}^{\alpha}+s_{1}^{\alpha}\right)}{\Gamma(\alpha+1)}\right] \| f\left(., x_{n}(.), B x_{n}(.)\right)-
$$
$f(., x(),. B x().) \|_{P C}$.

Case 3. For $t \in\left(s_{i}, t_{i+1}\right), i=1,2, \ldots, m$,

$$
\left\|Q_{2} x_{n}(t)-Q_{2} x(t)\right\| \leq\left[\frac{M_{A} t_{i+1}^{\alpha}+M_{A}^{2}\left(t_{i}^{\alpha}+s_{i}^{\alpha}\right)+\ldots+M_{A}^{i+1}\left(t_{1}^{\alpha}+s_{1}^{\alpha}\right)}{\Gamma(\alpha+1)}\right] \| f\left(., x_{n}(.), B x_{n}(.)\right)-
$$

$f(., x(),. B x().) \|_{P C}$.

Step4: $Q_{2}$ is equicontinuous, which means $\left\|Q_{2} x\left(t_{2}\right)-Q_{2} x\left(t_{1}\right)\right\| \rightarrow 0$ as $t_{2} \rightarrow t_{1}$.

For $0 \leq t^{\prime}<t^{\prime \prime} \leq t_{1}$, we have:

$$
\begin{aligned}
\left\|Q_{2} x\left(t^{\prime \prime}\right)-Q_{2} x\left(t^{\prime}\right)\right\| \leq & \| \int_{0}^{t^{\prime \prime}}\left(t^{\prime \prime}-s\right)^{\alpha-1} Q_{\alpha}\left(t^{\prime \prime}-s\right) f(s, x(s), B x(s)) d s \\
& -\int_{0}^{t^{\prime}}\left(t^{\prime}-s\right)^{\alpha-1} Q_{\alpha}\left(t^{\prime \prime}-s\right) f(s, x(s), B x(s)) d s \| \\
\leq & I_{1}+I_{2}+I_{3},
\end{aligned}
$$

where $I_{1}=\left\|\int_{t^{\prime}}^{t^{\prime \prime}}\left(t^{\prime \prime}-s\right)^{\alpha-1} Q_{\alpha}\left(t^{\prime \prime}-s\right) f(s, x(s), B x(s)) d s\right\|$,

$$
\begin{aligned}
& I_{2}=\left\|\int_{0}^{t^{\prime}}\left(t^{\prime}-s\right)^{\alpha-1}\left[Q_{\alpha}\left(t^{\prime \prime}-s\right)-Q_{\alpha}\left(t^{\prime}-s\right)\right] f(s, x(s), B x(s)) d s\right\|, \\
& I_{3}=\left\|\int_{0}^{t^{\prime}}\left[\left(t^{\prime \prime}-s\right)^{\alpha-1}-\left(t^{\prime}-s\right)^{\alpha-1}\right] Q_{\alpha}\left(t^{\prime \prime}-s\right) f(s, x(s), B x(s)) d s\right\|, \\
& I_{1} \leq \frac{\alpha M_{A}}{\Gamma(\alpha+1)} \int_{t^{\prime}}^{t^{\prime \prime}}\left\|\left(t^{\prime \prime}-s\right)^{\alpha-1} f(s, x(s), B x(s))\right\| d s \\
& \leq \frac{M_{A} S_{\alpha, \tau}}{\Gamma(\alpha)}\left(t^{\prime \prime}-t^{\prime}\right)^{\alpha-\tau} \longrightarrow 0 \text { as } t^{\prime \prime}-t^{\prime} \longrightarrow 0, \\
& I_{1} \longrightarrow 0 \text { as } t^{\prime \prime}-t^{\prime} \longrightarrow 0 \text {. }
\end{aligned}
$$

For $t^{\prime}=0,0<t^{\prime \prime}<t_{1}$, it is easy to see that $I_{2}=0$.

For $t^{\prime}>0$ and $\epsilon>0$ small enough, we have 


$$
\begin{aligned}
I_{2} \leq & \left\|\int_{0}^{t^{\prime}-\epsilon}\left(t^{\prime}-s\right)^{\alpha-1}\left[Q_{\alpha}\left(t^{\prime \prime}-s\right)-Q_{\alpha}\left(t^{\prime}-s\right)\right] f(s, x(s), B x(s)) d s\right\| \\
& +\left\|\int_{t^{\prime}-\epsilon}^{t^{\prime}-\epsilon}\left(t^{\prime}-s\right)^{\alpha-1}\left[Q_{\alpha}\left(t^{\prime \prime}-s\right)-Q_{\alpha}\left(t^{\prime}-s\right)\right] f(s, x(s), B x(s)) d s\right\| \\
\leq & \sup _{s \in\left[0, t^{\prime}-\epsilon\right]}\left\|Q_{\alpha}\left(t^{\prime \prime}-s\right)-Q_{\alpha}\left(t^{\prime}-s\right)\right\| \int_{0}^{t^{\prime}-\epsilon}\left\|\left(t^{\prime}-s\right)^{\alpha-1} f(s, x(s), B x(s))\right\| d s \\
& +\frac{2 M_{A}}{\Gamma(\alpha)} \int_{t^{\prime}-\epsilon}^{t^{\prime}}\left\|\left(t^{\prime}-s\right)^{\alpha-1} f(s, x(s), B x(s))\right\| d s \\
\leq & S_{\alpha, \tau}\left(t^{\prime \frac{\alpha-\tau}{1-\tau}}-\epsilon^{\frac{\alpha-\tau}{1-\tau}}\right)^{1-\tau} \sup _{s \in\left[0, t^{\prime}-\epsilon\right]}\left\|Q_{\alpha}\left(t^{\prime \prime}-s\right)-Q_{\alpha}\left(t^{\prime}-s\right)\right\|+\frac{2 M_{A}}{\Gamma(\alpha)} S_{\alpha, \tau} \epsilon^{\alpha-\tau}
\end{aligned}
$$

$I_{2} \longrightarrow 0$ as $t^{\prime \prime}-t^{\prime} \longrightarrow 0$ and $\epsilon \longrightarrow 0$.

$$
\begin{aligned}
I_{3} & \leq \int_{0}^{t^{\prime}}\left\|\left[\left(t^{\prime \prime}-s\right)^{\alpha-1}-\left(t^{\prime}-s\right)^{\alpha-1}\right] Q_{\alpha}\left(t^{\prime \prime}-s\right) f(s, x(s), B x(s))\right\| d s \\
& \leq \frac{M_{A} S_{\alpha, \tau}}{\Gamma(\alpha)}\left(\left(t^{\prime \prime}-t^{\prime}\right)^{\frac{\alpha-\tau}{1-\tau}}+t^{\prime \frac{\alpha-\tau}{1-\tau}}+t^{\prime \prime \frac{\alpha-\tau}{1-\tau}}\right)^{1-\tau} \\
& \leq \frac{M_{A} S_{\alpha, \tau}}{\Gamma(\alpha)}\left(t^{\prime \prime}-t^{\prime}\right)^{\alpha-\tau} \longrightarrow 0 ; t^{\prime \prime}-t^{\prime} \longrightarrow 0
\end{aligned}
$$

$I_{3} \longrightarrow 0$ as $t^{\prime \prime}-t^{\prime} \longrightarrow 0$.

Case 1. For $t_{i} \leq t^{\prime}<t^{\prime \prime} \leq s_{i}$,

$\left\|Q_{2} x\left(t^{\prime \prime}\right)-Q_{2} x\left(t^{\prime}\right)\right\|=0$.

Case 2. For $s_{i} \leq t^{\prime}<t^{\prime \prime} \leq t_{i+1}$,

$$
\left\|Q_{2} x\left(t^{\prime \prime}\right)-Q_{2} x\left(t^{\prime}\right)\right\| \leq I_{1}+I_{2}+I_{3}+\left\|\left(P_{\alpha}\left(t^{\prime \prime}-s_{i}\right)-P_{\alpha}\left(t^{\prime}-s_{i}\right)\right) K_{i 2 x}\right\| .
$$

The right-hand side of (4.1) tends to 0 independently of $x \in B_{r}$ as $t^{\prime \prime} \rightarrow t^{\prime}$.

Case 3. For $t_{i} \leq t^{\prime}<s_{i}<t^{\prime \prime} \leq t_{i+1}$,

$\left\|Q_{2} x\left(t^{\prime \prime}\right)-Q_{2} x\left(t^{\prime}\right)\right\| \leq \| P_{\alpha}\left(t^{\prime \prime}-s_{i}\right) K_{i 2 x}+\int_{0}^{t^{\prime \prime}}\left(t^{\prime \prime}-s\right)^{\alpha-1} Q_{\alpha}\left(t^{\prime \prime}-s\right) f(s, x(s), B x(s)) d s-$ $d_{i 2 x} \| \longrightarrow 0$

indenpendently of $x \in B_{r}$, as $t^{\prime \prime} \longrightarrow t^{\prime}$ we have $\left(t^{\prime \prime} \longrightarrow s_{i}\right)$.

In conclusion, $\left\|Q_{2} x\left(t^{\prime \prime}\right)-Q_{2} x\left(t^{\prime}\right)\right\| \longrightarrow 0$, as $t^{\prime \prime}-t^{\prime} \longrightarrow 0$, which implies that $Q_{2}\left(B_{r}(J)\right)$ is equicontinuous.

We have $Q_{2} B_{r} \subseteq B_{r}$, where $Q_{2} B_{r}(t)=\left\{Q_{2} x(t) ; x \in B_{r}\right\}$ for $t \in J$.

Step5: $Q$ is $\beta$ - condensing in $B_{r}$.

For any $W \subset B_{r}, Q_{2}(W)$ is bounded and equicontinuous. Hence, by lemma(2.10), there exists a countable set $W_{1}=\left\{u_{n}\right\}_{n=1}^{\infty} \subset W$ such that $\beta\left(Q_{2}(W)\right) \leq 2 \beta\left(Q_{2}\left(W_{1}\right)\right)$. Since $Q_{2}\left(W_{1}\right) \subset B_{r}$ is equicontinuous, lemma $(2.11)$ implies $Q_{2}\left(W_{1}\right)=\max \beta Q_{2}\left(W_{1}(t)\right)$. 
We have: $\beta\left(\left\{\int_{0}^{t} B(t, s) u(s) d s / u \in B_{r}, t \in J\right\}_{n=1}^{\infty}\right) \leq \omega K_{0} \beta\left(\left\{u(t) / u \in B_{r}, t \in J\right\}_{n=1}^{\infty}\right)$.

Case1. For $t \in\left[0, t_{1}\right]$, we have:

$$
\begin{aligned}
\beta\left(Q_{2}\left(W_{1}(t)\right)\right) & =\beta\left(\left\{\int_{0}^{t}(t-s)^{\alpha-1} Q_{\alpha}(t-s) f\left(s, u_{n}(s), B u_{u}(s)\right) d s\right\}_{n=1}^{\infty}\right) \\
& \leq \frac{2 M_{A}}{\Gamma(\alpha)} \int_{0}^{t}(t-s)^{\alpha-1} \beta\left(\left\{f\left(s, u_{n}(s), B u_{n}(s)\right)\right\}_{n=1}^{\infty}\right) d s \\
& \leq \frac{2 M_{A}}{\Gamma(\alpha)} \int_{0}^{t}(t-s)^{\alpha-1}\left(L_{1} \beta\left(W_{1}(s)\right)+L_{2} \beta\left(B\left(W_{1}\right)(s)\right)\right) d s \\
& \leq \frac{2 M_{A} L_{1}}{\Gamma(\alpha)} \int_{0}^{t}(t-s)^{\alpha-1} \beta\left(W_{1}(s)\right) d s+\frac{2 M_{A} L_{2} \omega K_{0}}{\Gamma(\alpha)} \int_{0}^{t}(t-s)^{\alpha-1} \beta\left(W_{1}(s)\right) d s \\
& \leq \frac{2 M_{A}\left(L_{1}+\omega L_{2} K_{0}\right) t_{1}^{\alpha}}{\Gamma(\alpha+1)} \beta(W) .
\end{aligned}
$$

Since $Q_{2}\left(W_{1}\right)$ is bounded and equicontinuous, by lemma (2.11)

$$
\begin{aligned}
\beta\left(Q_{2}(W)\right) & \leq 2 \beta\left(Q_{2}\left(W_{1}\right)\right)=2 \max _{t \in J} \beta\left(Q_{2}\left(W_{1}(t)\right)\right) \\
& \leq \frac{4 M_{A}\left(L_{1}+\omega L_{2} K_{0}\right) t_{1}^{\alpha}}{\Gamma(\alpha+1)} \beta(W) \\
& <\beta(W) .
\end{aligned}
$$

In the other hand we have:

$\left\|Q_{1} x(t)-Q_{1} y(t)\right\|=0$ which implies that $\beta\left(Q_{1}(W)\right)=0$.

Then

$$
\begin{aligned}
\beta(Q(W)) & \leq \beta\left(Q_{1}(W)\right)+\beta\left(Q_{2}(W)\right) \\
& \leq \frac{4 M_{A}\left(L_{1}+\omega L_{2} K_{0}\right) t_{1}^{\alpha}}{\Gamma(\alpha+1)} \beta(W)<\beta(W) .
\end{aligned}
$$

Case2. For $t \in\left(t_{i}, s_{i}\right], i=1,2, \ldots, m$, we have:

$\left\|Q_{1} x(t)-Q_{1} y(t)\right\| \leq\left[\frac{\left(s_{i}^{\beta}+t_{i}^{\beta}\right)+M_{A}\left(s_{i-1}^{\beta}+t_{i-1}^{\beta}\right)+\ldots+M_{A}^{i-1}\left(s_{1}^{\beta}+t_{1}^{\beta}\right)}{\Gamma(\beta+1)}\right] K\|x-y\|_{P C}$.

Hence, by definition (2.7) we get:

$$
\beta\left(Q_{1}(W)\right) \leq\left[\frac{\left(s_{i}^{\beta}+t_{i}^{\beta}\right)+M_{A}\left(s_{i-1}^{\beta}+t_{i-1}^{\beta}\right)+\ldots+M_{A}^{i-1}\left(s_{1}^{\beta}+t_{1}^{\beta}\right)}{\Gamma(\beta+1)}\right] K \beta(W) .
$$

On the other hand:

$$
\beta\left(Q_{2}(W)\right) \leq 4 M_{A}\left(L_{1}+\omega L_{2} K_{0}\right)\left[\frac{t_{i}^{\alpha}+M_{A}\left(t_{i-1}^{\alpha}+s_{i-1}^{\alpha}+\ldots+M_{A}^{i-1}\left(t_{1}^{\alpha}+s_{1}^{\alpha}\right)\right.}{\Gamma(\alpha+1)}\right] \beta(W) .
$$

Then 


$$
\begin{aligned}
\beta(Q(W)) \leq & \beta\left(Q_{1}(W)\right)+\beta\left(Q_{2}(W)\right) \\
\leq & {\left[\frac{\left(s_{i}^{\beta}+t_{i}^{\beta}\right)+M_{A}\left(s_{i-1}^{\beta}+t_{i-1}^{\beta}\right)+\ldots+M_{A}^{i-1}\left(s_{1}^{\beta}+t_{1}^{\beta}\right)}{\Gamma(\beta+1)}\right] K \beta(W) } \\
& +4 M_{A}\left(L_{1}+\omega L_{2} K_{0}\right)\left[\frac{t_{i}^{\alpha}+M_{A}\left(t_{i-1}^{\alpha}+s_{i-1}^{\alpha}\right)+\ldots+M_{A}^{i-1}\left(t_{1}^{\alpha}+s_{1}^{\alpha}\right)}{\Gamma(\alpha+1)}\right] \beta(W) \\
< & \beta(W) .
\end{aligned}
$$

Case3. For $t \in\left[s_{i}, t_{i+1}\right], i=1,2, \ldots, m$ we have:

$\left\|Q_{1} x(t)-Q_{1} y(t)\right\| \leq\left[\frac{M_{A}\left(s_{i}^{\beta}+t_{i}^{\beta}\right)+M_{A}^{2}\left(s_{i-1}^{\beta}+t_{i-1}^{\beta}\right)+\ldots+M_{A}^{i}\left(s_{1}^{\beta}+t_{1}^{\beta}\right)}{\Gamma(\beta+1)}\right] K\|x-y\|_{P C}$.

Hence, by definition (2.7) we get:

$$
\beta\left(Q_{1}(W)\right) \leq\left[\frac{M_{A}\left(s_{i}^{\beta}+t_{i}^{\beta}\right)+M_{A}^{2}\left(s_{i-1}^{\beta}+t_{i-1}^{\beta}\right)+\ldots+M_{A}^{i}\left(s_{1}^{\beta}+t_{1}^{\beta}\right)}{\Gamma(\beta+1)}\right] K \beta(W) .
$$

On the other hand:

$$
\beta\left(Q_{2}(W)\right) \leq 4 M_{A}\left(L_{1}+\omega L_{2} K_{0}\right)\left[\frac{t_{i+1}^{\alpha}+M_{A}\left(t_{i}^{\alpha}+s_{i}^{\alpha}\right)+\ldots+M_{A}^{i}\left(t_{1}^{\alpha}+s_{1}^{\alpha}\right.}{\Gamma(\alpha+1)}\right] \beta(W) .
$$

Then

$$
\begin{aligned}
\beta(Q(W)) & \leq \beta\left(Q_{1}(W)\right)+\beta\left(Q_{2}(W)\right) \\
& \leq\left[\frac{M_{A}\left(s_{i}^{\beta}+t_{i}^{\beta}\right)+M_{A}^{2}\left(s_{i-1}^{\beta}+t_{i-1}^{\beta}\right)+\ldots+M_{A}^{i}\left(s_{1}^{\beta}+t_{1}^{\beta}\right)}{\Gamma(\beta+1)}\right] K \beta(W) \\
& +4 M_{A}\left(L_{1}+\omega L_{2} K_{0}\right)\left[\frac{t_{i+1}^{\alpha}+M_{A}\left(t_{i}^{\alpha}+s_{i}^{\alpha}+\ldots+M_{A}^{i}\left(t_{1}^{\alpha}+s_{1}^{\alpha}\right)\right.}{\Gamma(\alpha+1)}\right] \beta(W) \\
& <\beta(W) .
\end{aligned}
$$

Conclusion: in all cases we have:

$$
\beta(Q(W)) \leq \beta\left(Q_{1}(W)\right)+\beta\left(Q_{2}(W)\right) \leq C \beta(W)<\beta(W) \text { with } C>0 .
$$

Since the operator $Q$ is continuous and $\beta$-condensing. According to DarboSadovskii's fixed point theorem, $Q$ has a fixed point in $B_{r}$. Therefore, the problem(1.2) has at least one mild solution in $B_{r}$. This completes the proof.

Theorem 4.2. Assume that $\left(H_{1}\right)-\left(H_{4}\right)$, and $\left(H_{5}^{\prime}\right)-\left(H_{6}\right)$ hold. In addition, let's suppose that the following property is verified:

$$
\max \{C, D\}<1
$$


Where $C=4 M_{A}\left(L_{1}+\omega L_{2} K_{0}\right)\left[\frac{t_{m}^{\alpha}+M_{A}\left(t_{m-1}^{\alpha}+s_{m-1}^{\alpha}\right)+\ldots+M_{A}^{m-1}\left(t_{1}^{\alpha}+s_{1}^{\alpha}\right)}{\Gamma(\alpha+1)}\right]$,

and $D=4 M_{A}\left(L_{1}+\omega L_{2} K_{0}\right)\left[\frac{t_{m+1}^{\alpha}+M_{A}\left(t_{m}^{\alpha}+s_{m}^{\alpha}\right)+\ldots+M_{A}^{m}\left(t_{1}^{\alpha}+s_{1}^{\alpha}\right)}{\Gamma(\alpha+1)}\right]$.

Then the problem (1.2) has at least one mild solution.

Proof. We introduce the composition $Q=Q_{1}+Q_{2}$ where :

$$
\begin{gathered}
Q_{1} x(t)=\left\{\begin{array}{c}
P_{\alpha}(t) x_{0}+\int_{0}^{t}(t-s)^{\alpha-1} Q_{\alpha}(t-s) C(s) u(s) d s, t \in\left[0, t_{1}\right], \\
d_{i 1 x}+\frac{1}{\Gamma(\beta)} \int_{0}^{t}(t-s)^{\beta-1} g_{i}(s, x(s)) d s, t \in\left(t_{i}, s_{i}\right], i=1,2, . ., m, \\
P_{\alpha}\left(t-s_{i}\right) K_{i 1 x}+\int_{0}^{t}(t-s)^{\alpha-1} Q_{\alpha}(t-s) C(s) u(s) d s, \\
t \in\left(s_{i}, t_{i+1}\right], i=1,2, . ., m,
\end{array}\right. \\
Q_{2} x(t)=\left\{\begin{array}{r}
\int_{0}^{t}(t-s)^{\alpha-1} Q_{\alpha}(t-s) f(s, x(s), B x(s)) d s, t \in\left[0, t_{1}\right], \\
d_{i 2 x}, t \in\left(t_{i}, s_{i}\right], i=1,2, . ., m, \\
P_{\alpha}\left(t-s_{i}\right) K_{i 2 x}+\int_{0}^{t}(t-s)^{\alpha-1} Q_{\alpha}(t-s) f(s, x(s), B x(s)) d s, \\
t \in\left(s_{i}, t_{i+1}\right], i=1,2, . ., m,
\end{array}\right.
\end{gathered}
$$

with

$$
\left\{\begin{aligned}
d_{i 1 x}= & P_{\alpha}\left(t_{i}-s_{i-1}\right) K_{(i-1) 1 x}+\int_{0}^{t_{i}}\left(t_{i}-s\right)^{\alpha-1} Q_{\alpha}\left(t_{i}-s\right) C(s) u(s) d s \\
& -\frac{1}{\Gamma(\beta)} \int_{0}^{t_{i}}\left(t_{i}-s\right)^{\beta-1} g_{i}(s, x(s)) d s, i=1,2, . ., m, \\
K_{i 1 x}= & d_{i 1 x}+\frac{1}{\Gamma(\beta)} \int_{0}^{s_{i}}\left(s_{i}-s\right)^{\beta-1} g_{i}(s, x(s)) d s \\
& -\int_{0}^{s_{i}}\left(s_{i}-s\right)^{\alpha-1} Q_{\alpha}\left(s_{i}-s\right) C(s) u(s) d s, i=1,2, . ., m, \\
K_{01 x}= & x_{0},
\end{aligned}\right.
$$

and

$$
\left\{\begin{array}{l}
d_{i 2 x}=P_{\alpha}\left(t_{i}-s_{i-1}\right) K_{(i-1) 2 x}+\int_{0}^{t_{i}}\left(t_{i}-s\right)^{\alpha-1} Q_{\alpha}\left(t_{i}-s\right) f(s, x(s), B x(s)) d s, i=1,2, . ., m, \\
K_{i 2 x}=d_{i 2 x}-\int_{0}^{s_{i}}\left(s_{i}-s\right)^{\alpha-1} Q_{\alpha}\left(s_{i}-s\right) f(s, x(x), B x(s)) d s, i=1,2, . ., m, \\
K_{02 x}=0 .
\end{array}\right.
$$

Our proof will be divided into several steps.

Step1: We show that $Q B_{r}(J) \subset B_{r}(J)$,

where $B_{r}=\{x \in P C(J, X) ;\|x\| \leq r\}$ the ball with radius $r>0$;

$K_{\alpha, \tau}=\alpha\left(\frac{1-\tau}{\alpha-\tau}\right)^{1-\tau}\|C u\|_{L^{1 / \tau}}$ and $S_{\alpha, \tau}=\alpha\left(\frac{1-\tau}{\alpha-\tau}\right)^{1-\tau}\|m\|_{L^{1 / \tau}}$, 


$$
\begin{aligned}
\gamma_{1} & =M_{A}^{m+1}\left\|x_{0}\right\| \frac{\Gamma(\beta+1)}{\Gamma(\beta+1)-M_{A} K\left(t_{1}^{\beta}+s_{1}^{\beta}\right)}, \\
\gamma_{2} & =\frac{\left(M_{A} t_{m+1}^{\alpha-\tau}+M_{A}^{2}\left(t_{m}^{\alpha-\tau}+s_{m}^{\alpha-\tau}\right)+\ldots+M_{A}^{m+1}\left(t_{1}^{\alpha-\tau}+s_{1}^{\alpha-\tau}\right)\right) \Gamma(\beta+1)}{\left(\Gamma(\beta+1)-M_{A} K\left(t_{1}^{\beta}+s_{1}^{\beta}\right)\right) \Gamma(\alpha)}\left(K_{\alpha, \tau}+\right.
\end{aligned}
$$

$\left.S_{\alpha, \tau}\right)$

$$
\gamma_{3}=\frac{M_{A}^{2}\left(t_{m-1}^{\beta}+s_{m-1}^{\beta}\right)+\ldots+M_{A}^{m}\left(t_{1}^{\beta}+s_{1}^{\beta}\right)}{\left(\Gamma(\beta+1)-M_{A} K\left(t_{1}^{\beta}+s_{1}^{\beta}\right)\right)} d .
$$

Here $\gamma_{1}+\gamma_{1}+\gamma_{1} \leq r$.

For any $x \in B_{r}$, we have:

Case1. For $t \in\left[0, t_{1}\right]$

$$
\begin{aligned}
\|Q x(t)\| & \leq\left\|P_{\alpha}(t) K_{0 x}\right\|+\left\|\int_{0}^{t}(t-s)^{\alpha-1} Q_{\alpha}(t-s)[f(s, x(s), B x(s))+C(s) u(s)] d s\right\| \\
& \leq M_{A}\left\|x_{0}\right\|+\frac{M_{A} t_{1}^{\alpha-\tau}}{\Gamma(\alpha)}\left(K_{\alpha, \tau}+S_{\alpha, \tau}\right) .
\end{aligned}
$$

Similar to the proof of the previous theorem we show that: for $t \in\left(t_{i}, s_{i}\right]$

$$
\begin{aligned}
\|Q x(t)\| \leq & M_{A}^{i}\left\|x_{0}\right\|+\frac{M_{A} t_{i}^{\alpha-\tau}+M_{A}^{2}\left(t_{i-1}^{\alpha-\tau}+s_{i-1}^{\alpha-\tau}\right)+\ldots+M_{A}^{i}\left(t_{1}^{\alpha-\tau}+s_{1}^{\alpha-\tau}\right)}{\Gamma(\alpha)}\left(K_{\alpha, \tau}+S_{\alpha, \tau}\right) \\
& +\frac{\left(t_{i}^{\beta}+s_{i}^{\beta}\right)+M_{A}\left(t_{i-1}^{\beta}+s_{i-1}^{\beta}\right)+\ldots+M_{A}^{i-1}\left(t_{1}^{\beta}+s_{1}^{\beta}\right)}{\Gamma(\beta+1)}(b\|x\|+d) \\
\leq & r .
\end{aligned}
$$

For $t \in\left(s_{i}, t_{i+1}\right]$

$$
\begin{aligned}
\|Q x(t)\| \leq & M_{A}^{i+1}\left\|x_{0}\right\|+\frac{M_{A} t_{i+1}^{\alpha-\tau}+M_{A}^{2}\left(t_{i}^{\alpha-\tau}+s_{i}^{\alpha-\tau}\right)+\ldots+M_{A}^{i+1}\left(t_{1}^{\alpha-\tau}+s_{1}^{\alpha-\tau}\right)}{\Gamma(\alpha)}\left(K_{\alpha, \tau}+S_{\alpha, \tau}\right) \\
& +\frac{M_{A}\left(t_{i}^{\beta}+s_{i}^{\beta}\right)+M_{A}^{2}\left(t_{i-1}^{\beta}+s_{i-1}^{\beta}\right)+\ldots+M_{A}^{i}\left(t_{1}^{\beta}+s_{1}^{\beta}\right)}{\Gamma(\beta+1)}(b\|x\|+d) \\
\leq & r .
\end{aligned}
$$

We proved that $Q B_{r}(J) \subset B_{r}(J)$.

Step2: $Q_{2}$ is continuous.

Let $\left(x_{n}\right)_{n \geq 0}$ be a sequence such that $\lim _{n \rightarrow \infty}\left\|x_{n}-x\right\|_{P C}=0$, we have by $\left(H_{5}^{\prime}\right)$ : $g_{i}\left(t, x_{n}(t)\right) \longrightarrow g_{i}(t, x(t))$.

Case 1. For $t \in\left[0, t_{1}\right]$, we have:

$\left\|Q_{1} x_{n}(t)-Q_{1} x(t)\right\|=0$

Case 2. For $t \in\left[t_{i}, s_{i}\right], 1 \leq i \leq m$, 
$\left\|Q_{1} x(t)-Q_{1} y(t)\right\| \leq\left[\frac{\left(s_{i}^{\beta}+t_{i}^{\beta}\right)+M_{A}\left(s_{i-1}^{\beta}+t_{i-1}^{\beta}\right)+\ldots+M_{A}^{i-1}\left(s_{1}^{\beta}+t_{1}^{\beta}\right)}{\Gamma(\beta+1)}\right]\left\|g_{i}\left(t, x_{n}(t)\right)-g_{i}(t, x(t))\right\|_{P C}$.

For $t \in\left[s_{i}, t_{i+1}\right], 1 \leq i \leq m$,

$\left\|Q_{1} x(t)-Q_{1} y(t)\right\| \leq\left[\frac{M_{A}\left(s_{i}^{\beta}+t_{i}^{\beta}\right)+M_{A}^{2}\left(s_{i-1}^{\beta}+t_{i-1}^{\beta}\right)+\ldots+M_{A}^{i}\left(s_{1}^{\beta}+t_{1}^{\beta}\right)}{\Gamma(\beta+1)}\right]\left\|g_{i}\left(t, x_{n}(t)\right)-g_{i}(t, x(t))\right\|_{P C}$.

Thus we get $\left\|Q_{1} x_{n}(t)-Q_{1} x(t)\right\|_{P C} \longrightarrow 0$ as $n \longrightarrow \infty$.

Then we can say that $Q_{1}$ is continuous.

We already have that $Q_{2}$ is continuous. Finally $Q$ is continuous.

Step3: $Q$ is $\beta$ - condensing in $B_{r}$.

Case1. For $t \in\left[0, t_{1}\right]$, we have:

Considering the condition $\left(H_{5}^{\prime}\right)$ and using the same method in the previous theorem we get:

$$
\begin{aligned}
\beta(Q(W)) & \leq \beta\left(Q_{2}(W)\right) \\
& \leq \frac{4 M_{A}\left(L_{1}+\omega L_{2} K_{0}\right) t_{1}^{\alpha}}{\Gamma(\alpha+1)} \beta(W) \\
& <\beta(W) .
\end{aligned}
$$

Case2. For $t \in\left(t_{i}, s_{i}\right], i=1,2, \ldots, m$, we have:

$$
\begin{aligned}
\beta(Q(W)) & \leq \beta\left(Q_{2}(W)\right) \\
& \leq 4 M_{A}\left(L_{1}+\omega L_{2} K_{0}\right)\left[\frac{t_{i}^{\alpha}+M_{A}\left(t_{i-1}^{\alpha}+s_{i-1}^{\alpha}\right)+\ldots+M_{A}^{i-1}\left(t_{1}^{\alpha}+s_{1}^{\alpha}\right)}{\Gamma(\alpha+1)}\right] \beta(W) .
\end{aligned}
$$

Case3: For $t \in\left[s_{i}, t_{i+1}\right], i=1,2, \ldots, m$, we have:

$$
\begin{aligned}
\beta(Q(W)) & \leq \beta\left(Q_{2}(W)\right) \\
& \leq 4 M_{A}\left(L_{1}+\omega L_{2} K_{0}\right)\left[\frac{t_{i+1}^{\alpha}+M_{A}\left(t_{i}^{\alpha}+s_{i}^{\alpha}\right)+\ldots+M_{A}^{i}\left(t_{1}^{\alpha}+s_{1}^{\alpha}\right)}{\Gamma(\alpha+1)}\right] \beta(W) .
\end{aligned}
$$

Conclusion: in all cases we have:

$\beta(Q(W)) \leq \beta\left(Q_{2}(W)\right) \leq C \beta(W)<\beta(W)$ with $C>0$.

Since the operator $Q$ is continuous and $\beta$-condensing. According to DarboSadovskii's fixed point theorem, $Q$ has a fixed point in $B_{r}$. Therefore, the prob$\operatorname{lem}(1.2)$ has at least one mild solution in $B_{r}$. This completes the proof. 


\section{Authors CONTRIButions}

All authors contributed equally to the writing of this paper. All authors read and approved the final manuscript.

\section{REFERENCES}

[1] Benchohra.M , Henderson, J.Ntouyas, SK. Impulsive Differential Equations and inclusions. Hindawi publishing, New York(2006).

[2] P. Chen, Y. Li, Monotone iterative technique for a class of semilinear evolution equations with nonlocal conditions, Results Math, 63(2013)731-744.

[3] P. Chen, X. Zhang, Y. Li. Existence of mild solutions to partial differential equations with non-instantenous impulses. Electronic Journal of Differential Equations, vol. 2016(2016), No. 241, pp. 1-11.

[4] M.M. El-Borai, Some probability densities and fundamental solutions of fractional evolution equations, Chaos solitons fractals 14(2002)433-440.

[5] M.M. El-Borai, Semigroups and some nonlinear fractional differential equations, Appl.Math; Comput. 149(2004)823-831.

[6] Peter L. Falb, Infinite Dimensional control problems: On the closure of the set of attainable states for linear systems, Mathematical Analysis and Application 9,12-22(1964).

[7] Gou,M, Xue, X. Li. R. Controllability of impulsive evolution inclusions with nonlocal conditions. J. Optim. Theory Appl 120,255-374(2004).

[8] Banas,J Goebel, Measure of noncompactness in banach space Lecture notes in Pure and Applied Mathematics, Vol60, Marcel Dekker, New york (1980).

[9] R. Hilfer, Apllications of Fractional Calculus in Physics, World Scientific, Singapore(2000).

[10] H.P Heinz, on the behaviour of measure of noncompactness with respect to differentiation and integration of vector-valued functions, Nonlinear Anal. 7(1983)1351-1371.

[11] A.A. Kilbas, H.M. Srivastava, J.J. Trujillo, Theory and Applications of Fractional Differential Equations, North-Holland Mathematics Studies, vol, 204, Elsevier, Amsterdam, 2006.

[12] P. Kumar, D. Pandey, D. bahuguna. On a new class of abstract impulsive functional differential equations of fractional order, J. Nonlinear Sci. Appl. 7(2014). 102-114.

[13] V. Lakshmikantham, Theory of fractional functional differential equations, Nonlinear Anal. (2007), doi:10.1016/j.na.2007.09.025.

[14] V. Lakshmikantham, A.S. Vatsala, Basic theory of fractional differential equations, Nonlinear Anal. (2007),doi:10.1016/j.na.2007.09.025.

[15] Lakshmikantham. V,Bainov. DD, Simeonov. PS. Theory of impulsive Differential Equations. World Scientific, Singapore(1989).

[16] K.S. Miller, B.Ross, An introduction to the fractional Calulus nad Fractional Differential Equations, Wiley, New York, 1993.

[17] I.Podlubny, Fractional Differential Equations, Academic Press, New York, 1993.

[18] A. Pazy, Semigroups of linear Operators and Applications to partial Differential Equations, Springer-Verlag, Berlin 1983.

[19] J. Wang, M. Feckan and Y. Zhou, On the new concept of solutions and existence results for impulsive fractional evolution equations, Dyn. Partial Differ. Equ. 8(2011), 345-361.

[20] J. Wang, Y. Zhou, A class of fractional evolution equations and optimal controls, Nonlinear Anal. RWA 12(2011)262-272.

[21] Xi Fu, Xiaoyou Liu, Bowen Lu: On a new class of impulsive fractional evolution equations. Advances in Difference Equations, vol. 2015, article 227,2015.

[22] Yong Zhou, Basic theory of fractional differential equations, Xiangtan University, China, 2014. 
(K. Hilal) Department of Mathematics, Faculty of Sciences and Technics, Sultan Moulay Slimane University, BP 523 Beni Mellal 23000, Morocco

E-mail address, K. Hilal: khalid.hilal.usms@gmail.com

(K. Guida) Department of Mathematics, Faculty of Sciences and Technics, Sultan Moulay Slimane University, BP 523 Beni Mellal 23000, Morocco

E-mail address, K. Guida: guida.karim@gmail.com

(L. Ibnelazyz) Department of Mathematics, Faculty of Sciences and Technics, Sultan Moulay Slimane University, BP 523 Beni Mellal 23000, Morocco

E-mail address, L. Ibnelazyz: ibnelazyzlahcen@gmail.com

(M. Oukessou) Department of Mathematics, Faculty of Sciences and Technics, Sultan Moulay Slimane University, BP 523 Beni Mellal 23000, Morocco

E-mail address, M. Oukessou: ouk.mohamed@yahoo.fr 